\title{
Auto Regressive lonospheric Prediction model for GPS Applications
}

\author{
Karthik.P, D. Venkata Ratnam, Nagireddy Vanga, P. S. Brahmanadam, \\ B. Sada Siva Rao and K. Sarat Kumar \\ Dept of ECE,K L University ,Vaddeswaram, Guntur Dt, Andhra Pradesh, India.
}

\begin{abstract}
Global Positioning System (GPS) is a satellite based navigation system. The GPS system positional accuracy is limited by ionospheric error. Ionospheric delay is a function of Total Electron Content (TEC). Prediction of ionospheric delays is very important for high precision application such as civil aircraft landing and missile guidance application. In this paper, a new modeling technique known as an Auto Regressive (AR) model is proposed for predicting the TEC values. This model is based on short term time series analysis. This model works with basic principle of regression, where past data i.e. Previous days TEC is used for predicting future values. The GPS data by Hyderabad station are considered. It is found that the AR model gives better results for short term predictions.
\end{abstract}

\section{Keywords}

Ionospheric TEC, prediction, AR model, regression, forecast

\section{INTRODUCTION}

The total electron content (TEC) is an important parameter in measuring variation of ionospheric layer, which affects the radio transmissions from satellite to earth [1]. TEC is a measure of electron content along the radio propagation with a square meter area cross section. In radio propagation there are many errors in which ionospheric errors play a major role due to large variations of TEC movement over the F-layer region, due to these movements accumulations of electrons may vary which depends on seasonal changes, atmospheric conditions, landscapes and sudden disturbances [2,3,4]. TEC variations of a dual frequency Global Positioning System (GPS) provide an overall description of Ionospheric layer, which can be used to determine time delays, range error corrections and also used for forecasting of future TEC variations.

As the need of navigation and precise positioning has vast applications became more prominent in nowadays like aerospace communication, aircraft landing marine navigation etc. They need accurate data points with future variation. There are many models for forecasting future TEC variations in which Auto Regressive (AR) model is more reliable than other models [5]. This model has an accuracy of 70\% 80\% in predicting future TEC values. The time series analysis has been widely used in the forecasting field such as weather forecast, market prediction, earthquake forecasting, autocontrol, and so on, while it has been rarely applied to the study of the ionospheric characteristics [6,7]. Time series analysis theory and technology of a new model have been developed and is used to analyze a new ionospheric TEC prediction technique using autoregressive models (AR) to perform short-term regional ionospheric TEC prediction. The predicted TEC are compared with the TEC measured by International GNSS services (IGS) stations, and with the TEC derived from model is analyzed to check the performance of the AR model developed for forecasting [8].

\section{AUTO REGRESSIVE MODEL}

Auto Regressive (AR) model works as a univariate time domain estimation of the spectral density function and a linear predictor of a stationary time series objects like statistics and signal processing $[9,10]$. The main function of this model is forecasting the future values based on past and present values in the series. AR model part has an order factor by which smoothen the filter output responses and it has all-pole filter response characteristics, because all pole filter is more sensitive to variations in input and minimum prediction error. In certain events cases lower order gives better results than higher order cases and data must be a time series, continuity, and stationary data.

The previous GPS TEC data by Hyderabad station consider as $\{\mathrm{x}(\mathrm{t})\}(\mathrm{t}=1,2, \ldots, \mathrm{N})$ where $\mathrm{N}$ is total previous days and $\mathrm{t}$ gives particular previous day. Time series data obtained is not stationary because of seasonal changes, climate and system changes, so data is transformed to stationary time series values by regular differencing the values before predicting [11]. The randomness of the object is given by

$$
\mathrm{X}_{\mathrm{t}}=\mathrm{X}_{\mathrm{t}-1}+\mathrm{Z}_{\mathrm{t}}
$$

Where $Z_{t}=W N\left(0, \sigma^{2}\right)$ is the white noise with zero mean and constant covariance $\left(\sigma^{2}\right)$

The equation below explains the basic AR equation with regression coefficients $\left(\emptyset_{\mathrm{k}}\right)$, variance $(\mu)$, error factor $\left(\epsilon_{t-1}\right)$ and past time series values $\left(x_{t}\right)$.The polynomial for the AR model is given by

$x_{t}=a_{1} x_{t-1}+a_{2} x_{t-2}+\ldots+a_{p} x_{t-p}+\epsilon_{t}$

Where $a_{1}, a_{2}, a_{3}, a_{4}, \ldots . a_{p}$ were AR coefficients, $\epsilon_{t}$ is white noise with zero mean and constant covariance, $\mathrm{p}$ is the order of the AR model and $x_{t-1}$ terms are stationary time series data.

The first order and second order terms are analyzed given by

$$
\begin{aligned}
& x_{t-1}-\mu=a\left(x_{t}-\mu\right)+\epsilon_{t-1} \\
& x_{t-1}-\mu=a_{1}\left(x_{1}-\mu\right)+a_{2}\left(x_{t-2}-\mu\right)+\epsilon_{t+1}
\end{aligned}
$$

$\mu$ is the mean value of the original time series of VTEC data, the coefficients of the autoregressive model are developed by the matrix method, for which autocovarience of stationary time series samples is computed as

$C_{x x}(l)=\frac{1}{N} \sum_{n=1}^{N} X(n+l) X(n)$

If $\mathrm{p}$ is the order of the autoregressive model $(\mathrm{p}=1,2,3 \ldots l)$ then $a_{1}, a_{2}, a_{3}, a_{4}, \ldots . a_{p}$ parameters are determined by matrix given as 


$$
\left[\begin{array}{cccccc}
1 & r_{1} & r_{2} & r_{3} & \ldots & r_{n-1} \\
r_{1} & 1 & r_{1} & r_{2} & \ldots & r_{n-2} \\
r_{2} & r_{1} & 1 & r_{1} & \ldots & r_{n-3} \\
r_{3} & r_{2} & r_{1} & 1 & \ldots & r_{n-4} \\
\vdots & \vdots & \vdots & \vdots & \vdots & \vdots \\
r_{n-1} & r_{n-2} & r_{n-3} & r_{n-4} & \ldots & 1
\end{array}\right] *\left[\begin{array}{c}
a_{1} \\
a_{2} \\
a_{3} \\
a_{4} \\
\vdots \\
a_{n}
\end{array}\right]=\left[\begin{array}{c}
r_{1} \\
r_{2} \\
r_{3} \\
r_{4} \\
\vdots \\
r_{n}
\end{array}\right]
$$

Autocorrelation is applied to the data collected, later regression analysis is implemented and Lag operations are used to get clear results.

\section{DATA COLLECTION}

The TEC data of dual frequency GPS receiver's data from Hyderabad station is obtained from this analysis from Scripps Orbit and Permanent Array Centre (SOPAC) where data is collected from 2 local IGS stations near the equator in India which are, Hyderabad (HYDE) receiver (geographic $17.41 \mathrm{~N}$ Lat, $78.55^{\circ} \mathrm{E}$ Long, geomagnetic $8.26^{\circ} \mathrm{N}$ late) and Bangalore (IISC) receiver (geographic $13.02 \mathrm{~N}$ Lat, $77.57^{\circ} \mathrm{E}$ Long, geomagnetic $4.38^{\circ} \mathrm{N}$ lat). 16 day data from 1 st Jan 2012 to 16th Jan 2012 and 19th of Feb 2012 and 212st of FEB 2012 are also used for this analysis, data are available in RINEX (Receiver Independent Exchange) format [12].

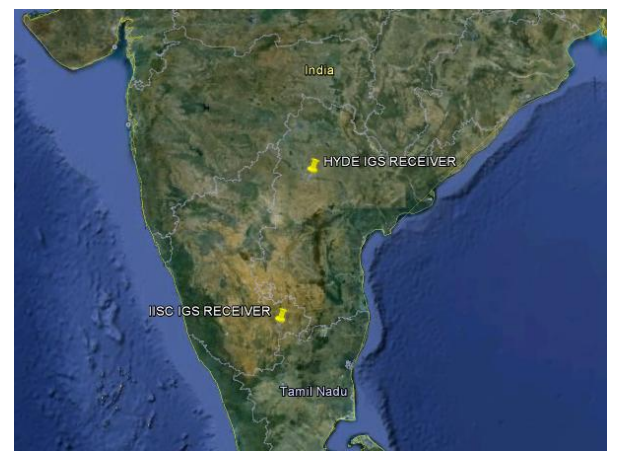

Figure-1: Two IGS receiver stations HYDE and IISC in India

RINEX data are converted to data files having GPS week, time, elevation angle, azimuthal angle, Slant TEC, Vertical TEC, latitude, longitude. The obtained VTEC of all days are used for predicting the future TEC values.

\section{RESULTS AND DICUSSIONS}

VTEC trends of 9 days (1st Jan 2012 to 9th Jan 2012) are presented in Fig-2. The sampling rate of the trends is 6 minutes. It is observed from the figure is that, time series of TEC data showing diurnal behavior. This time series of VTEC data will be applied to the AR model for forecasting ionospheric TEC values. The TEC values have reached a maximum of 70 TEC units on the day of (2nd Jan 2012).

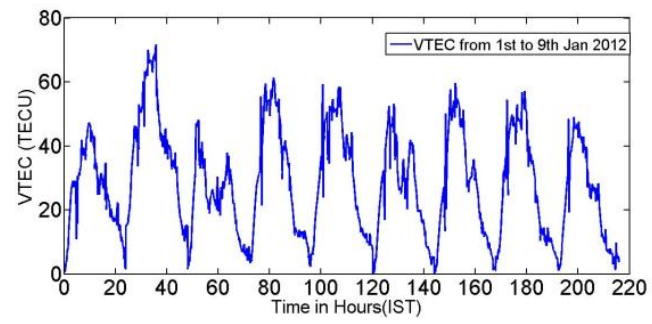

Figure-2: VTEC Original data trends of 1st-9th Jan 2012 from HYDE, India receiver
Using AR model coefficients are derived which are used on the 9 days data to forecast next seven days. The forecasted values are compared with actual TEC values to determine the accuracy of the prediction in Fig-3 (a). Due to large disturbances on 3rd day of TEC prediction (on 12th Jan 2012) has a large prediction error. The error in predicting future trends will gives the behavior of AR model in predicting future VTEC trends. In initial day prediction trends, prediction error is low after determining further day future trends the error in prediction decreased. The corresponding prediction error values are presented in Fig-3 (b). The prediction error varies between 20TECU to -30TECU.
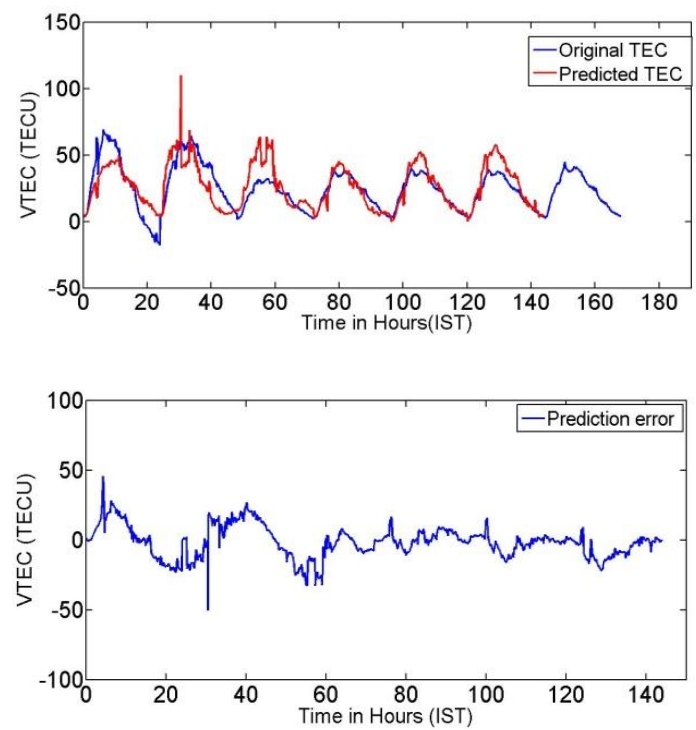

Figure-3: (a) Comparison between original VTEC to predicted VTEC trends, (b) Error in prediction of HYDE receiver

The performance of AR model is investigated on a quiet day (21st Feb 2012, KP index max-4) and disturbed day (19th Feb 2012, KP index max-6) in the fig- 4 (C), 5 (C). In order to forecast TEC values 5 days (16th Feb 2012 to 20th Feb 2012) are considered, corresponding forecasted data of the quiet day and disturbed day are shown in Fig-4 (a) and Fig-5 (a) and TEC variations in the original data of disturbed day has varied up to 88TECU and for normal day it is varied up to 68TECU. From the prediction error measurement, the disturbed day has a variation of $12 \mathrm{ECU}$ to $-28 \mathrm{TECU}$ and for quiet day the variations ranges from 18 TECU to -5 TECU. It is observed that TEC values due to disturbed day have more prediction error than quiet day from the fig-4 (b), 5 (b).

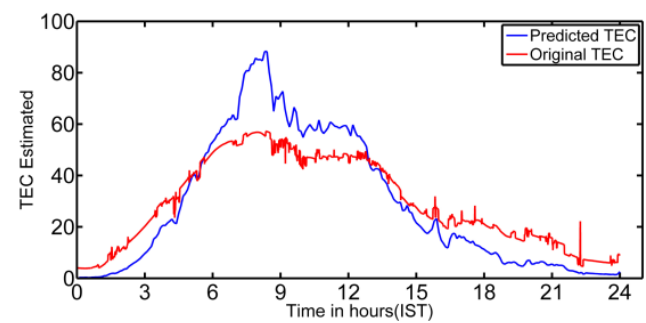



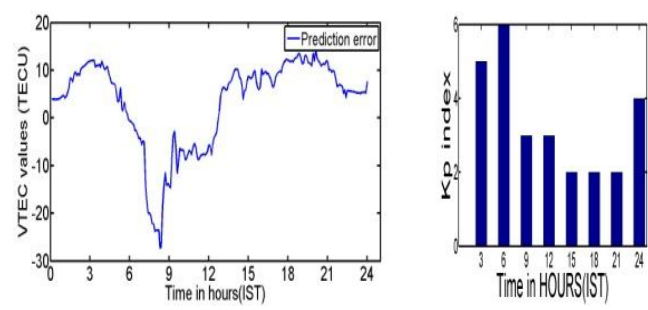

Figure-4:(a) Disturbed day on 19th Feb 2012 w r t Kp index factor, original VTEC trend(red line), predicted VTEC trend(blue line), (b) Prediction error with original VTEC data of a Disturbed day on 19th Feb 2012 and (c) Kp index on 19th Feb 2012
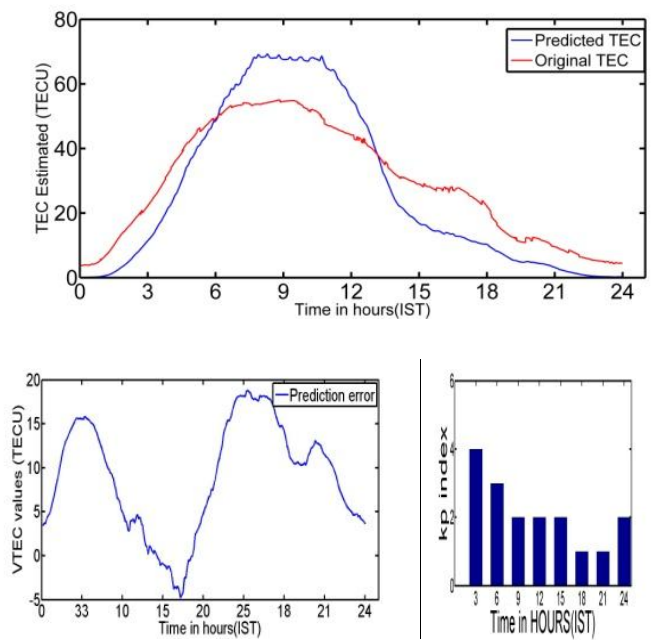

Figure-5: (a)Normal day on 21st Feb 2012 w $r$ t Kp index factor, original VTEC trend(red line), predicted VTEC trend(blue line), (b) Prediction error with original VTEC data of a normal day on 21st Feb 2012 and (c) Kp index on

21st Feb 2012

\section{CONCLUSIONS}

Autoregressive is the most effective model for analyzing time series data. Ionospheric TEC variations are high in low latitude regions, for that an effective AR model is used for forecasting the time series VTEC variation. Regression analysis is mainly used for predicting future trends of TEC values. By using 9 days Hyderabad TEC data are used for predicting next 6 days TEC data. It is observed that the initial prediction error is high at the beginning of the time series of VTEC data. It is evident that the forecasted values due to AR model are closely following the actual VTEC measurements. These preliminary results indicated that, AR model is able to perform well in both disturbed in quiet day conditions. By using seasonal, atmospheric conditions and magnetic behavior of earth will helps in accurate prediction of TEC

\section{ACKNOWLEDGEMENT}

The above work has been carried out under the project entitled "Development of Ionospheric Forecasting models for Satellite based Navigation Systems over low latitude stations" sponsored by Department of Science and Technology, New Delhi, India, vide sanction letter No: SR/FTP/ETA0029/2012, dated: 08.05.12.

\section{REFFERENCES}

[1] P. M. Kintner, B. M. Ledvina, and E. R. de Paula, 'GPS and ionospheric scintillations", SPACE WEATHER, VOL. 5, pages 260-283, 10th Oct 2007.

[2] Z. G. Elmas, M. Aquino, H. A. Marques, and J. F. G. Monico, ":Higher order ionospheric effects in GNSS positioning in the European region", Ann. Geophys., 29, 1383-1399, 2011.

[3] W. Dean Pesnell, 'PREDICTIONS OF SOLAR CYCLE 24", Solar Cycle 24, May 242007.

[4] Clynch, J.R. (2001) "Ionospheric Bending of Radio Signals: A rough, Shell Model Approximation", published as part of Professor Clynch's collection of technical notes available athttp://clynchg3c.com/Technote/Tnotes.htm.

[5] McLeod, A. I. Zhang, "Partial Autocorrelation parameterization auto regression ", Journal of time series analysis $27,599-612,2006$.

[6] Kleusberg, A, "The Global Positioning System and Ionospheric Condition”, Solar Prediction-IN Workshop, Ottawa,Canada, NOAA/Dept. of Commerce Pub.,Vol.1,PP.142-146,1993.

[7] Doherty, P.H.,T Dehel, J.A.Klobuchar, S.H.Delay, S.Datta-Barua, E.R.de Paula and F.S. Rodrigues, "Ionospheric effects on low- latitude space based augmentation system," in proc. ION GPS ,2002, pp.1321-1329.

[8 ]S. Lu, K. H. Ju, and K. H. Chon, "A new algorithm for linear and non-linear ARMA model parameter estimation", IEEE Trans. Biomed. Eng., vol. 48, pp. 1116-1124, Oct. 2001.

[9] Li Zhi-Gang Cheng Zong-yi et all, "A study of prediction models for ionosphere", Chinese journal of geophysics, vol 50, no-2, pp 307 319, 2007.

[10] Hirotugu Akarine, "Fitting Auto Regressive models for prediction", Annals of the Institute of Statistical Mathematics, Springer, vol. 21(1), pages 225-242, December 1989.

[11] WANG Zhen-long,HU Yong-hong, "Time series analysis and its application, " Science Press,Beijing,2007.

[12] GPS RINEX data is available from the link below and it is maintained by US department of Defense to store GPS data for free access to public use www.sopac.ucsd.edu/cgiin/dbDataBySite.cgi 